\title{
Optical Trapping of Magnetotactic Bacteria and Effect of Magnetic Field on
}

\section{Trapping Force}

Vivek S. Jadhav $^{\text {a }}$, Srikanya Kundu ${ }^{\mathrm{b}}$, Arun G. Banpurkar, Gauri R. Kulkarni ${ }^{\mathrm{a}}$

${ }_{b}^{a}$ Department of Physics, University of Pune, Pune 411007, India.

School of Basic Medical Science, University of Pune, Pune 411007, India.

Cell movement is a topic of interest, using optical tweezers and magnetotactic bacteria (MTB) having an intracellular chain of magnetic nanoparticles, magnetosomes, is a fast growing field of scientists interest. Motion of MTB under the influence of magnetic field, optical trapping force required for single MTB and influence of external static magnetic field on a trapped MTB are relatively less explored aspects. In the present work, using single beam optical tweezer, we describe a first experiment with Magnetospirillum magnetotacticum (MS-1) trapping and its motion under the influence of magnetic field is observed and quantified. Motion of MTB within magnetic fields is influenced by the chain of magnetosomes. This opens the possibility to study the dynamics of MTB in confined volume under the influence of external magnetic field.

References

[1] R.B. Frankel et al., Biophys. J. 73 (1997) 994-1000.

[2] M. Tanase et al., Lab Chip. 5 (2005) 598-605.

[3] H. Lee et al., Nano Lett. 4 (2004) 995-998.

[4] J.D. Beck et al., Nano Lett. 5 (2005) 777-781.

[5] M. Hakoda et al., Biotechnol. Prog. 21 (2005) 1748-1753.

[6] A. Krichevsky et al., J. Appl. Phys. 101 (2007) 014701-014706

[7] K. Erglis et al., Biophys. J. 93 (2007) 1402-1412.

[8] G.V. Soni et al., Biophys. J. 84 (2003) 2634-2637.

[9] S. Chattopadhyay et al., PNAS, 103 (2006) 13712-13717.

[10] N.C. Darnton et al, J. Bact., 189 (2007) 1756-1764.

[11] S. Kundu et al., Biomaterials, 30 (2009). 4211-4218. 

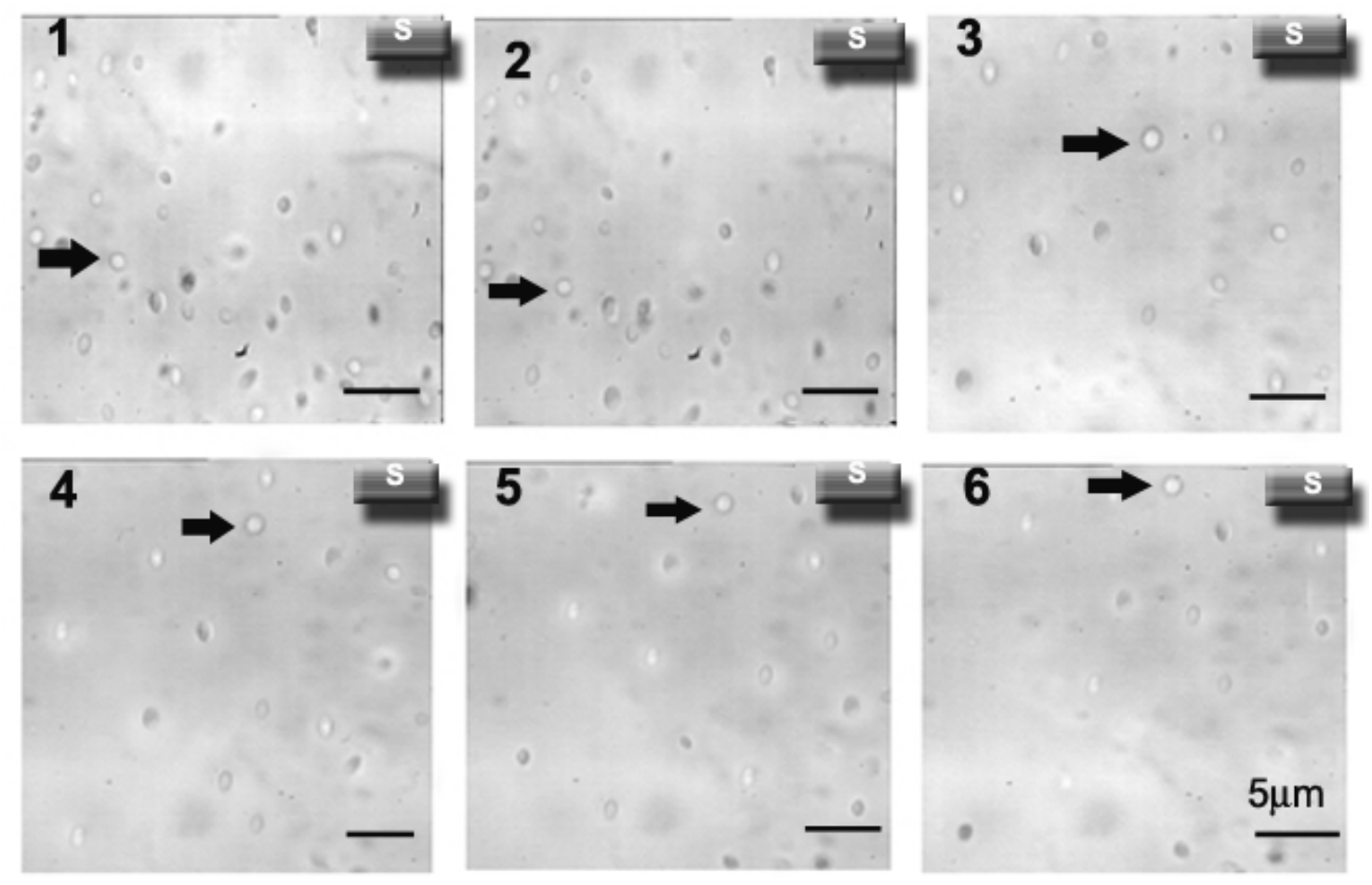

Fig 1: Real time imaging of trapped MTB in presence of external static magnetic field. Black arrow point out the observed cell.

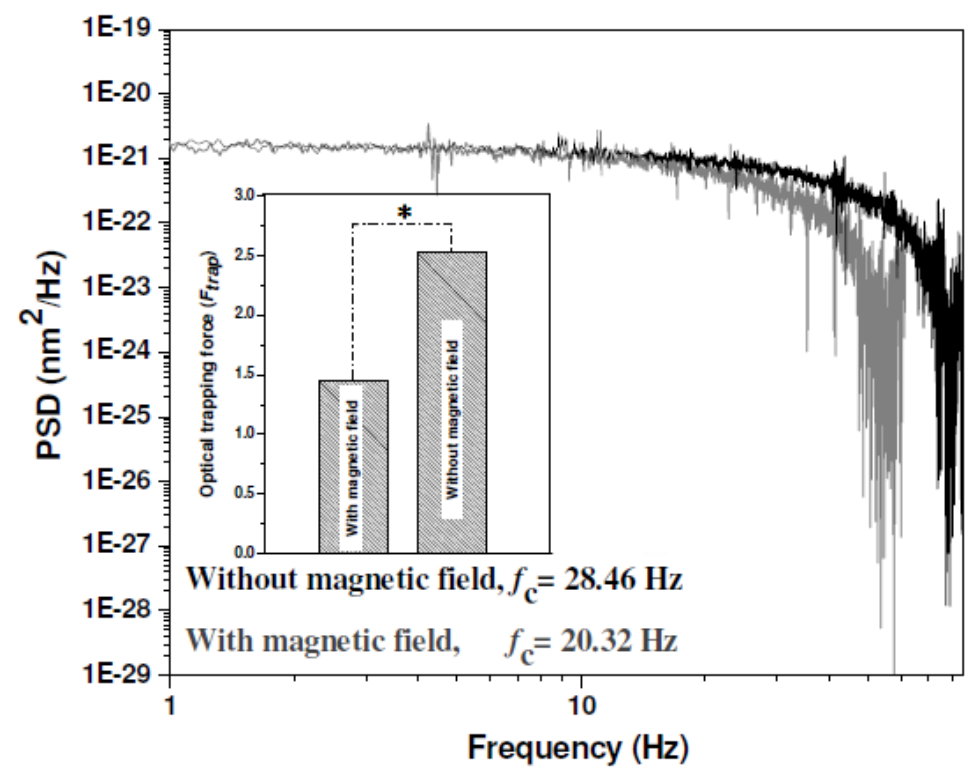

Fig 2: The power spectrum density (PSD) of trapped magnetotactic bacterium fluctuations at $0.75 \mathrm{~W}$ output laser power trap, with and without the presence of external static magnetic filed (67 G). Inset: Comparative optical force plot. 